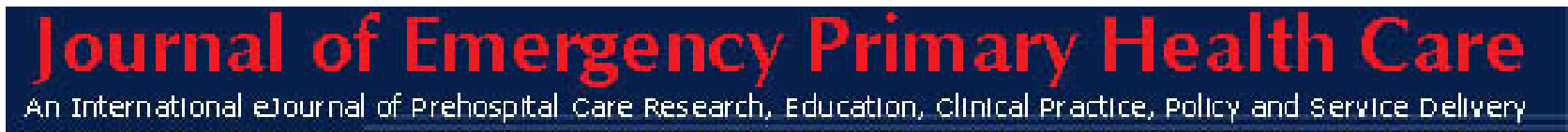

1447-4999

\title{
EDITORIAL
}

\section{PEER REVIEW AND JEPHC}

Article No. 990064

Frank Archer

An essential requirement of a profession's scientific journal is an effective and transparent process of peer review. With the exception of editorials, book and web site reviews, ACAP Updates, product reviews, conference reports and profiles, all articles offered to JEPHC are peer reviewed.

A peer review process has three main functions: to ensure that submitted articles meet the scope and editorial policies of the particular journal; to assess that the article is likely to be of interest to the readers of the journal; and, to ensure that the article meets accepted professional, scientific, and ethical standards. It is not the purpose of peer review to census or to argue or disagree with the context, argument or outcome of the article. The peer review process respects the rights of the author(s) to explain their study and/or opinions in their own way.

The peer review process for JEPHC is outlined on this web site in the Reviewer guidelines but is worth repeating here and commenting on the experience gained in the first two editions of JEPHC.

When articles are received by the editorial assistant, they are entered on a tracking system and deidentified. An internal review is undertaken by one of the Editorial team to assess if the article meets the Author guidelines of JEPHC. If the article meets these guidelines, the editorial team member nominates two external reviewers for the professional, scientific and ethical review. As a principle, the external reviewers are chosen from two different states other than that of the principle author of the article under review. At least one external reviewer is always a paramedic with qualifications, experience and/or interest in the topic of the particular article. The second reviewer will depend on the nature of the article. Sometimes a third reviewer may also be used. Articles submitted by MUCAPS staff are always referred to a member of the editorial team from outside MUCAPS for the internal review and for nomination of external reviewers, who will also be from outside Victoria.

In the past year, 59 mixed articles have been submitted to JEPHC, 32 of which were assessed by members of the editorial team as being suitable for peer review. Following successful completion of the peer review process, 13 articles have since been accepted for publication, 3 articles were voluntarily withdrawn by their authors during the review process, and 9 are pending revision by their authors following recommendations provided by reviewers. Three articles are currently in the initial phase of the peer review process. Only 4 were initially rejected by the reviewers as not being suitable for publication in JEPHC - reasons being that the articles had either been published in other journals, were of marginal interest to potential readers of JEPHC, or the presentation was grossly outside the editorial policies of JEPHC.

It may be of interest to potential authors to note that no article was accepted for publication without recommendation by the reviewers for amendments to be considered by the authors. 25 articles were returned to authors with recommendations for consideration of which 13 were amended, re-submitted and subsequently published. 


\section{Journal of Emergency Primary Health Care (JEPHC), Vol.1, Issue3-4, 2003}

The peer review process takes approximately 4-6 weeks and is undertaken by double blind review, meaning that authors and reviewers have no details of one another. The reviewers are provided with a standardised evaluation proforma which is returned to the authors in due course. The process of peer review is managed by Rhona Macdonald.

The JEPHC editorial team recognises that preparing a paper for publication is not undertaken lightly, nor is it an easy task. However, if the field of Emergency Primary Health Care is to advance, those involved in this important area of health care should be encouraged to share their experiences with others, be they substantiated opinions, professional experiences or the outcome of structured evaluations or research. The scope and major styles of articles encouraged by JEPHC are outlined in the Author guidelines.

Because JEPHC is new and many potential authors may be inexperienced in preparing articles for publication, JEPHC offers support and guidance to those potential authors. In the first instance, contact should be made with the Editorial Assistant, Rhona Macdonald. Rhona can provide initial guidance on editorial policies and procedures and can arrange for other members of the editorial team to provide advice and guidance on content and presentation style. Potential authors are encouraged to access this free and willing advice.

In this issue, at the end of Volume 1, the editorial team would like to acknowledge the expert assistance provided by the 30 peer reviewers who have helped shape the first volume of JEPHC. A list of these reviewers is included in this edition. Their contribution is warmly acknowledged.

If other readers would like to become peer reviewers for JEPHC, then they should forward an expression of interest, including their name, contact details, brief $\mathrm{CV}$, and fields of interest, by email to $\underline{\text { Rhona Macdonald. }}$

The editorial team looks forward to expanding the panel of peer reviewers, expanding the number of articles, and improving the quality of JEPHC. 